\title{
Author index (Vol. 10)
}

Abbasi, M., Golchin, A. \& Saany, H. M., Characterization of monoids by condition $(G P)$

Abdulnaby, Z. E., see Ibrahim

Afshari, H. \& Kheiryan, A., Tripled fixed point theorems and applications to a fractional differential equation boundary value problem

Alam, N. \& Khan, N. M., Epimorphisms, dominions, closed and absolutely closed semigroups

Alhevaz, A., Baghipur, M. \& Rahimi, S., Bounds on hyperWiener index of graphs

Ali, A. \& Othman, W. A. M., Geometric aspects of CRwarped product submanifolds of $T$-manifolds

Ali, A., see Shafique

Alibemani, A. \& Hashemi, E., The total graph of unfaithful submodules of a module over reversible rings

Ali Choudhary, M., Totally real submanifolds in a generalized complex space form

Alizadeh, B., see Modabber

Alizadeh, S. \& Moradlou, F., Weak and strong convergence theorems and a nonlinear ergodic theorem for $N$ generalized hybrid mappings

Angadi, L. M., see Shiralashetti

Ankur Self-dual codes over the ring $G R\left(p^{m}, g\right)$ and Jacobi forms

Arabyani, H., see Saeedi

Argyros, I. K. \& George, S., Local convergence of deformed Euler-Halley-type methods in Banach space under weak conditions

10 (2017) 1750086

Awasthi, A., see Kadalbajoo

10 (2017) 1750021

10 (2017) 1750075

Azimi, M. \& Moussavi, A., On nilpotent elements of ore extensions

10 (2017) 1750029

10 (2017) 1750043

Baghipur, M., see Alhevaz

Banakh, T. O. \& Gavrylkiv, V. M., On structure of the semi-

10 (2017) 1750056 groups of $k$-linked upfamilies on groups

Baroughi, F., see Modabber

Bell, H. E., Commutativity of rings with identities on subsets

10 (2017) 1750057

10 (2017) 1750062

10 (2017) 1750057

Botmart, T., Yotha, N., Mukdasai, K. \& Wongaree, S., Global synchronization for hybrid coupled neural networks with interval timevarying delays: A matrixbased quadratic convex approach

10 (2017) 1750025

Challab, K. A., Darus, M. \& Ghanim, F., Certain problems related to generalized Srivastava-Attiya operator

Chaudhari, J. N. \& Suryawanshi, M. D., On weakly $n$ absorbing subtractive ideals in semirings

10 (2017) 1750083

10 (2017) 1750017

10 (2017) 1750018

10 (2017) 1750027

10 (2017) 1750035

10 (2017) 1750017

10 (2017) 1750001

10 (2017) 1750026

10 (2017) 1750055 10 (2017) 1750076

Chiang, Y.-J., Equivariant exponentially harmonic maps between manifolds with metrics of signatures

Czerwik, S. \& Król, K., Fixed point theorems in generalized metric spaces

Darabi, A. \& Forouzanfar, A.-M., Periodic shadowing and standard shadowing property

Darus, M., see Challab
10 (2017) 1750060

10 (2017) 1750006

10 (2017) 1750063

10 (2017) 1750030

10 (2017) 1750027 
Dass, B. K., Sharma, N. \& Verma, R., Perfect codes in poset block spaces

Deshi, A. B., see Shiralashetti

Dipl. Math. (FH) Lange, K., On irrational Brun-type constants

Dutta, I., see Kar

El-Ashwah, R. M., see Ghanim

Fan, X., Teng, Z. \& Muhammadhaji, A., Global dynamics of a stochastic ratiodependent predator-prey system

Farsad, F. \& MoghbeliDamaneh, H., Weighted limits in the category Dcpo- $S$

Feng, M., see Lu

Firoozjaee, M. A., see Khorshidi

Forouzanfar, A.-M., see Darabi

Gala, S. \& Ragusa, M. A., On the regularity criterion for the Navier-Stokes equations in terms of one directional derivative

Gandhi, S. \& Ravichandran, V., Starlike functions associated with a lune

Ganesa Moorthy, C. \& Ramasamy, C. T., Pringsheim convergence of double sequences for uniform boundedness principle

Gavrylkiv, V. M., see Banakh

Gaynullina, A., On one class of commutative operads

George, S., see Argyros

Ghanim, F. \& El-Ashwah, R. M., Inclusion properties of certain subclass of univalent meromorphic functions defined by a linear operator associated with HurwitzLerch zeta function

Ghanim, F., see Challab

Golchin, A., see Abbasi

Gülsu, M., see Öztürk

Guo, Y. Q., Wang, S. F. \& Shum, K. P., Restricted Burnside problem for semigroups and its application to language theory
10 (2017) 1750005

10 (2017) 1750026

10 (2017) 1750079

10 (2017) 1750038

10 (2017) 1750066

10 (2017) 1750002

10 (2017) 1750037

10 (2017) 1750072

10 (2017) 1750031

10 (2017) 1750006

10 (2017) 1750012

10 (2017) 1750064

10 (2017) 1750080

10 (2017) 1750083

10 (2017) 1750007

10 (2017) 1750086

10 (2017) 1750066

10 (2017) 1750027

10 (2017) 1750021

10 (2017) 1750053

10 (2017) 1750045
Gupta, A. \& Sharma, P., Weighted fractional differentiation composition operators from mixed-norm spaces to Zygmund spaces

Gupta, R., see Thakur

Hamid, I. S. \& Saravanakumar, S., Open packing saturation number of a graph

Hashemi, E., see Alibemani

Hedayat Mahmoudi, M. \& Schulze, B.-W., Corner boundary value problems

Ibrahim, R. W., A. Kılıçman \& Abdulnaby, Z. E., Boundedness of fractional differential operator in complex spaces

Irannezhad, S. \& Madanshekaf, A., On classification of pomonoids by properties of generators

Ismael, M., Nillsen, R. \& Williams, G., Standard deviation of recurrence times for piecewise linear transformations

Izadi, M., see Zaeri

Jampachon, P., see Lohapan

Kadalbajoo, M. K. \& Awasthi, A., Parameter free hybrid numerical method for solving modified Burgers' equations on a nonuniform mesh

Kantli, M. H., see Shiralashetti

Kar, S. \& Dutta, I., Globally determined ternary semigroups

Khalil, S. \& Schulze, B.-W., Boundary problems on a manifold with edge

Khan, N. M., see Alam

Kheirfam, B., New complexity analysis of a full NesterovTodd step interior-point method for semidefinite optimization

Kheiryan, A., see Afshari

Khoeilar, R. \& Sheikholeslami, S. M., Rainbow reinforcement numbers in digraphs

Khorshidi, M. N., Yousefi, S. A. \& Firoozjaee, M. A., Determination of an unknown
10 (2017) 1750070

10 (2017) 1750082

10 (2017) 1750047

10 (2017) 1750033

10 (2017) 1750074

10 (2017) 1750054

10 (2017) 1750075

10 (2017) 1750041

10 (2017) 1750009

10 (2017) 1750071

10 (2017) 1750051

10 (2017) 1750029

10 (2017) 1750026

10 (2017) 1750038

10 (2017) 1750087

10 (2017) 1750062

10 (2017) 1750056

10 (2017) 1750004 
source term for an inverse source problem of the timefractional equation

Kılıçman, A., see Ibrahim

Kolivand, F., see Taghavi

Koppitz, J., see Susanti

Koşan, M. T. \& Quynh, T. C., On infinite direct sums of lifting modules

Król, K., see Czerwik

Kumar, P., see Prasad

Kumar, V. V. \& Rao, M. S., Dual annihilator filters of commutative $B E$-algebras

Lee, M., Symplectic diffeomorphisms with limit shadowing

Le Nam, T., Some results on curves in the plane with loglinear density

Liu, L., see Ma

Lohapan, L. \& Jampachon, P., Semigroup properties of linear terms

Lu, G. \& Feng, M., Positive solutions of $n$ th-order impulsive eigenvalue problems with an advanced argument

Ma, T., Liu, L. \& Xu, S., Twisted tensor biproduct monoidal Hom-Hopf algebras

Madanshekaf, A., see Irannezhad

Mahato, A. K., see Singh

Mishra, V. N. \& Sharma, P., Statistical approximation properties of modified Durrmeyer $q$ Baskakov type operators with two parameters $\alpha$ and $\beta$

Modabber, L., Alizadeh, B. \& Baroughi, F., A modified optimal algorithm for 2-maxian location problems on cactus graphs

Moghbeli-Damaneh, H., see Farsad

Mohammadi Aghjeh Mashhad, F., Gorenstein homological dimensions and some duality results

Moradi, S., Homological invariants of the Stanley-Reisner ring of a $k$-decomposable simplicial complex

\section{0 (2017) 1750031 \\ 10 (2017) 1750075 \\ 10 (2017) 1750044 \\ 10 (2017) 1750058}

10 (2017) 1750049

10 (2017) 1750030

10 (2017) 1750019

10 (2017) 1750013

10 (2017) 1750068

10 (2017) 1750085

10 (2017) 1750011

10 (2017) 1750051

10 (2017) 1750072

10 (2017) 1750011

10 (2017) 1750041

10 (2017) 1750042

10 (2017) 1750028

10 (2017) 1750017

10 (2017) 1750037

10 (2017) 1750048

10 (2017) 1750061
Moradlou, F., see Alizadeh

Moussavi, A., see Azimi

Moussavi, A., see Paykan

Moussavi, A., see Paykan

Muhammadhaji, A., see Fan

Mukdasai, K., see Botmart

Mursaleen, M. \& Nasiruzzaman, Md., Dunkl generalization of Kantorovich type Szász-Mirakjan operators via $q$-calculus

Nasiruzzaman, Md., see Mursaleen

Nigam, A., see Sharma

Nillsen, R., see Ismael

Niroomand, P., see Saeedi

Othman, W. A. M., see Ali

Öztürk, Y. \& Gülsu, M., Numerical solution of Abel equation using operational matrix method with Chebyshev polynomials

Patchakhieo, S. \& Pibaljommee, B., Characterizations of ordered $k$-regular semirings by ordered $k$-ideals

Patil, A. \& Waphare, B. N., On the zero-divisor graph of Rickart *-rings

Paykan, K. \& Moussavi, A., Nilpotent elements and nilArmendariz property of skew generalized power series rings

Paykan, K. \& Moussavi, A., The McCoy condition on skew monoid rings

Pelaitay, G., Discrete duality for 3-valued Łukasiewicz-Moisil algebras

Pibaljommee,

B.,

see Patchakhieo

Pinigina, N. R., On the wellposedness of boundary value problems for nonclassical differential equations of higher order

Pitchaimani, M. \& Ramesh Kumar, D., On Nadler type results in ultrametric spaces with application to wellposedness

Pitchaimani, M., see Ramesh Kumar

Prajapati, B., see Tiwari
10 (2017) 1750001

10 (2017) 1750043

10 (2017) 1750034

10 (2017) 1750050

10 (2017) 1750002

10 (2017) 1750025

10 (2017) 1750077

10 (2017) 1750077

10 (2017) 1750052

10 (2017) 1750009

10 (2017) 1750076

10 (2017) 1750067

10 (2017) 1750053

10 (2017) 1750020

10 (2017) 1750015

10 (2017) 1750034

10 (2017) 1750050

10 (2017) 1750003

10 (2017) 1750020

10 (2017) 1750059

10 (2017) 1750073

10 (2017) 1750065

10 (2017) 1750069 
Prasad, A. \& Kumar, P., Abelian theorems for fractional wavelet transform

Quynh, L. N., Algebraic dependences of meromorphic mappings sharing moving hyperplanes without counting multiplicities

Quynh, T. C., see Koşan

Ragusa, M. A., see Gala

Rahimi, S., see Alhevaz

Ramasamy, A. M. S., Diophantine quadruples and nearDiophantine quintuples from $P_{3, k}$ sequences

Ramasamy, C. T., see Ganesa Moorthy

Ramesh Kumar, D. \& Pitchaimani, M., Set-valued contraction mappings of Prešić-Reich type in ultrametric spaces

Ramesh Kumar, D., see Pitchaimani

Rao, M. S., see Kumar

Rasi, R. \& Sheikholeslami, S. M., The harmonic index of unicyclic graphs

Ravichandran, V., see Gandhi

Romano, D. A., Normally conjugative relations

Saany, H. M., see Abbasi

Saeedi, F., Arabyani, H. \& Niroomand, P., On dimension of Schur multiplier of nilpotent lie algebras II

Saeedi, H., see Zaeri

Saini, S., see Singh

Saravanakumar, S., see Hamid

Schulze, B.-W., see Hedayat Mahmoudi

Schulze, B.-W., see Khalil

Shafique, S. \& Ali, A., On the reduced second Zagreb index of trees

Sharma, N., see Dass

Sharma, P. \& Nigam, A., The Fekete-Szegö problem for a Ma-Minda type class of biunivalent functions associated with the Hohlov operator

Sharma, P., see Gupta

Sharma, P., see Mishra

Sharma, P., Faber polynomial coefficient estimates for a
10 (2017) 1750019

10 (2017) 1750040

10 (2017) 1750049

10 (2017) 1750012

10 (2017) 1750057

10 (2017) 1750010

10 (2017) 1750080

10 (2017) 1750065

10 (2017) 1750073

10 (2017) 1750013

10 (2017) 1750039

10 (2017) 1750064

10 (2017) 1750036

10 (2017) 1750021

10 (2017) 1750076

10 (2017) 1750071

10 (2017) 1750046

10 (2017) 1750033

10 (2017) 1750054

10 (2017) 1750087

10 (2017) 1750084 10 (2017) 1750005

10 (2017) 1750052

10 (2017) 1750082

10 (2017) 1750028 class of analytic bi-univalent functions involving a certain differential operator

10 (2017) 1750016

10 (2017) 1750069

10 (2017) 1750032

Sharma, R. K., see Yadav

Sheikholeslami, S. M., see Khoeilar

Sheikholeslami, S. M., see Rasi

Shiralashetti, S. C., Angadi, L. M., Kantli, M. H. \& Deshi, A. B., Numerical solution of parabolic partial differential equations using adaptive gird Haar wavelet collocation method

Shum, K. P., see Guo

Shum, K. P., see Singh

Singh, A. K., Mahato, A. K. \& Shum, K. P., Quasicoretractable modules

Singh, U. \& Saini, S., Approximation of periodic functions in certain sub-classes of $L^{p}[0,2 \pi]$

Suryawanshi, M. D., see Chaudhari

Susanti, Y. \& Koppitz, J., On endomorphisms of powersemigroups

Taghavi, A. \& Kolivand, F., Maps preserving strong 2Jordan product on some algebras

Taghavi, A., Maps preserving jordan triple product on the self-adjoint elements of $C^{*}$ algebras

Tărnăuceanu, M., On a generalization of the Gauss formula

Teng, Z., see Fan

Thakur, N. K., Gupta, R. \& Upadhyay, R. K., Complex dynamics of diffusive predator-prey system with Beddington-DeAngelis functional response: The role of prey-taxis

Thirupathi, G., On a subclass of quasi-convex functions with fixed second coefficient

Tiwari, S. K., Prajapati, B. \& Sharma, R. K., Banach algebra with generalized derivations

Tiwari, S. K., see Yadav
10 (2017) 1750069

10 (2017) 1750004

10 (2017) 1750039

10 (2017) 1750026

10 (2017) 1750045

10 (2017) 1750042

10 (2017) 1750042

10 (2017) 1750046

10 (2017) 1750063

10 (2017) 1750058

10 (2017) 1750044

10 (2017) 1750022

10 (2017) 1750008

10 (2017) 1750002

10 (2017) 1750047

10 (2017) 1750078

10 (2017) 1750032 
Udhayakumar, R., $n$-IFP-flat modules and $n$-IFP-injective modules

10 (2017) 1750014

Upadhyay, R. K., see Thakur

Verma, R., see Dass

Wang, S. F., see Guo

Waphare, B. N., see Patil

Williams, G., see Ismael

Wongaree, S., see Botmart

$\mathrm{Xu}, \mathrm{S}$., see $\mathrm{Ma}$

Yadav, V. K., Tiwari, S. K. \& Sharma, R. K., Generalized derivations on Lie ideals in prime rings

Yamaleev, R. M., Derivation of the system of generalized Riccati equations from

10 (2017) 1750047

10 (2017) 1750005

10 (2017) 1750015

10 (2017) 1750009

10 (2017) 1750025

10 (2017) 1750011

10 (2017) 1750032 the system of evolution equations

10 (2017) 1750081

Yotha, N., see Botmart

10 (2017) 1750025

10 (2017) 1750045

Yousefi, S. A., see Khorshidi

Zaeri, S., Saeedi, H. \& Izadi, M., Fractional integration operator for numerical solution of the integro-partial time fractional diffusion heat equation with weakly singular kernel

Zhao, R., Attached prime ideals of generalized inverse polynomial modules

10 (2017) 1750031

Zhou, F., A generalization of Hall's theorem
10 (2017) 1750071

10 (2017) 1750023

10 (2017) 1750024 